\title{
MIGRACIÓN Y MARGINALIDAD URBANA EN PAÍSES SUBDESARROLLADOS
}

\author{
Álvaro LóPEZ TORO \\ Universidad de los Andes, Bogotá
}

\section{INTRODUCCIÓN}

Este aRTículo trata de dar un paso en la exploración teórica de algunos fenómenos propios de la urbanización que actualmente experimentan muchos países en proceso de desarrollo, siguiendo la línea abierta recientemente en la literatura económica.1,2,3 Nuestro propósito es discutir las posibilidades de una tendencia del coeficiente de subempleo urbano hacia una posición de equilibrio y, alternativamente, introducir la consideración de algunas fuerzas que parecen incidir sobre el movimiento demográfico rural-urbano y que puede imprimir un ritmo explosivo al coeficiente de subempleo, bajo ciertas condiciones.

El punto de partida de Todaro en este sentido ha sido postular una hipótesis de dependencia de la tasa de migración rural-urbana con respecto a la diferencia relativa de los ingresos esperados, por parte de un migrante potencial, en el sector moderno urbano y el sector rural. Esta hipótesis elabora significativamente el supuesto previo de que la tasa de migración está regulada económicamente por el diferencial de ingresos entre las personas vinculadas a la agricultura y al sector avanzado de las ciudades, ${ }^{4}$ en la medida en que se introduce la influencia de la probabilidad de consecución de empleo en este último sector, como variable endógena del sistema que incide sobre la tasa migratoria. En las palabras de Todaro, "un $70 \%$ de premio en el salario real urbano, por ejemplo, podría ser de muy poca significación para un migrante en prospecto, si su probabilidad de conseguir empleo fuese, dijéramos, de uno en cincuenta". ${ }^{5}$

Para simplificar el problema, supondremos con Todaro que el horizonte de decisión del migrante es de un solo período. En este caso la tasa de inmigración $i$ en un período $t$, está dada por la expresión:

1 M. Todaro, "A Model of Labor Migration and Urban Unemployment in Less Developed Countries", American Economic Review, marzo de 1969.

2 M. Todaro, "An Analysis of Industrialization, Employment and Unemployment in Less Developed Countries", Yale Economic Essays, Otoño 1968, Vol. 8, Núm. 2.

3 C. R. Frank, Jr., "Urban Unemployment and Economic Growth in Africa", Oxford Economic Papers, julio de 1968.

4 Arthur Lewis, "Unemployment in Developing Countries", Midwestern Research Conference, octubre de 1964.

5 Todaro, Ref. 1, p. 140. 


$$
i(t)=F\left[\frac{\pi(t) Y_{u}(t)-Y_{r}(t)}{Y_{r}(t)}\right]
$$

siendo $Y_{r}(t)$ y $Y_{u}(t)$ el ingreso esperado por período en el campo y en la ciudad respectivamente, y $\pi(t)$ la probabilidad de conseguir el empleo en el sector moderno urbano, durante el período de migración $t$. Se supone que la correspondiente probabilidad para el campo es 1 . No vemos claramente cómo Todaro transforma ${ }^{6}$ la expresión (1) en

$$
i=\pi(t) F\left[\frac{Y_{u}(t)-Y_{r}(t)}{Y_{r}(t)}\right]
$$

En efecto, aparte de la falta de correspondencia algebraica entre (1) y (2), las dos ecuaciones expresan una influencia de $\pi(t)$ sobre $i$ sustancialmente diferente. De acuerdo con la ecuación (1), es posible para un valor bajo de $\pi(t)$ que $i$ resulte negativa aun con $Y_{u}(t)>Y_{r}(t)$, lo cual nunca ocurre en (1-a). Es decir, esta última ecuación no da cabida al fenómeno de migración urbana-rural cuando las oportunidades de empleo en la ciudad son muy reducidas, en contra de lo que el mismo autor ilustra ${ }^{7}$ con respecto a la existencia de tal fenómeno durante la depresión de los años treinta en Estados Unidos.

En seguida se comparan las implicaciones de una u otra formulación de la tasa inmigratoria sobre la dinámica del proceso de urbanización.

\section{EXISTENCIA Y ESTABILIDAD DE UNA TASA EQUILIBRADA DE INMIGRACIÓN}

Supongamos con Todaro que la fuerza laboral urbana crece a una tasa vegetativa $\beta$, uniforme de un período a otro, en tanto que la correspondiente tasa de generación de empleo en el sector moderno de la ciudad es $\gamma$, también constante. Siendo $N(t)$ y $S(t)$, respectivamente, el número de personas ocupadas en el sector urbano moderno y el total de la fuerza laboral urbana en el período $t$, la diferencia $S(t)-N(t)$ representará el número de personas desocupadas, más aquellas incorporadas al sector urbano tradicional y sometidas a un régimen de subocupación o de marginalidad económica. La proporción $E(t)$ de la fuerza laboral urbana ocupada en el sector moderno durante el período $t$, será

$$
E(t)=\frac{N(t)}{S(t)}
$$

en tanto que la probabilidad objetiva $\pi(t)$ de enganche al sector moderno para el mismo período será

$$
\pi(t)=\frac{\dot{N}(\mathrm{t})}{S(t)-N(t)}=\frac{\gamma N(t)}{S(t)-N(t)}=\frac{\gamma E(t)}{1-E(t)}
$$

bajo el supuesto de que todos los marginados aspiran y son elegibles para trabajar en las actividades modernas de avanzada. Es posible que esta probabilidad objetiva sea transitoriamente diferente de la

6 Todaro, Ref. 1, ecuación (8), p. 143.

7 Ibid., p. 140. 
probabilidad subjetiva $\pi^{\prime}(t)$ que rige la decisión migratoria en (1) o en (1-a), aunque de otra parte puede argumentarse que si existen discrepancias entre ambas probabilidades, no dejarán de manifestarse ciertos mecanismos de ajuste que tiendan a equipararlas. Por ejemplo, si $\pi^{\prime}(t)$ es mayor que $\pi(t)$, se presentará un sobreflujo de migrantes que no encontrará trabajo en el sector moderno de la ciudad, lo que hará bajar el valor de $E(t)$. Si existen señales adecuadas de información dentro del sistema, esta reducción de $E(t)$ no pasará desapercibida por los nuevos migrantes en perspectiva, con lo cual se produciría un reajuste de $\pi^{\prime}(t)$ en los próximos períodos. Se supondrá en lo que sigue, como una simplificación del problema, que $\pi(t)=\pi^{\prime}(t)$. Si se acepta además que los niveles de ingreso son constantes a lo largo del tiempo en el campo y en la ciudad, o que los diferenciales relativos permanecen sin variación de un período a otro, es decir, $Y_{u}(t)=x Y_{r}(t)$, $x>1$, entonces tendremos las siguientes expresiones para la tasa total de crecimiento de la población urbana:

Según la ecuación (1),

$$
\frac{S(t)}{S(t)}=\beta+F[\pi(t) x-1]=\beta+F\left[\gamma x \frac{E(t)}{1-E(t)}-1\right]
$$

Según la ecuación (1-a),

$$
\frac{\dot{S}(t)}{S(t)}=\beta+\pi(t) F(x-1)=\beta+\frac{\gamma E(t)}{1-E(t)} F(x-1)
$$

Para que el coeficiente de empleo esté en equilibrio se requiere

$$
\dot{E}(t)=\frac{d}{d t}[E(t)]=0
$$

Derivando ambos miembros de la ecuación (2) y sustituyendo (5) se tiene como una condición necesaria de equilibrio

$$
\begin{aligned}
& \gamma=\frac{\dot{N}}{N}(t)=\frac{\dot{S}}{S}(t) \\
& \gamma=\beta+F\left[\gamma x \frac{E^{* *}}{1-E^{* *}}-1\right] \\
& \gamma=\beta+\frac{\gamma E^{*}}{1-E^{*}} F(x-1)
\end{aligned}
$$

siendo $E^{*}$ el valor de equilibrio de $E$ en $(7-a)$ y $E^{* *}$ el valor de equilibrio en (7). Parece legítimo aceptar que $F(x)$ sea una función monotónica creciente de $x$, caso en el cual existe una función inversa monovalente $F^{-1}$ tal que $F^{-1}(F(x))=x$. En esta forma se puede resolver la la ecuación (7) para $E^{* *}$, obteniéndose 


$$
E^{* *}=\frac{1+F^{-1}(\gamma-\beta)}{1+F^{-1}(\gamma-\beta)+\gamma x}
$$

De otra parte, la solución de (7-a) conduce a

$$
E^{*}=\frac{\gamma-\beta}{(\gamma-\beta)+\gamma F(x-1)}
$$

Es conveniente ilustrar la diferencia del estimativo del valor de equilibrio a que conducen ambas fórmulas para el ejemplo propuesto por Todaro, 8 en el que $\gamma=.04, \beta=.02, x=2, F(x)=x$. Según la versión (8-a), $E^{*}=.33$, en tanto que de acuerdo con $(8), E^{* *}=.93$. Este último valor es excesivamente alto comparado con la situación actual de los centros urbanos en los países subdesarrollados, e induce a modificar algunas de las premisas del modelo migratorio. Este asunto será el objeto de la próxima sección.

Entre tanto, tratemos de dilucidar los aspectos de estabilidad del equilibrio definido por las ecuaciones (7) y (7-a). Todaro ${ }^{9}$ aborda este problema analizando el comportamiento de la variable $\frac{\dot{E}}{E}$, esto es, de la tasa de crecimiento de $E$ en una posición de perturbación con respecto al punto de equilibrio $E^{*}$, definido en (8-a) y, después de mostrar que la primera derivada de $\frac{\dot{E}}{E}$ con respecto a desplazamientos de la variable $E$, es negativa, infiere la estabilidad del equilibrio.

A este respecto, pueden hacerse dos observaciones. En primer lugar, si se procede de tal manera sólo se establece la aceleración o desaceleración de la oscilación de $E$ con respecto a $E^{*}$, pero no la inversión de la oscilación misma. Para lograr esto último, hay que determinar el comportamiento de $\frac{d E}{d t}$ partiendo de una perturbación con respecto a $E^{*}$. Es posible que la tasa de cambio, $\frac{d}{d E} \frac{\dot{E}}{E}$ sea negativa pero si $\frac{d E}{d t}$ se mantiene positiva para una posición de perturbación a la derecha del punto de equilibrio, no habrá estabilidad estática, que es la que analiza Todaro. En segundo lugar, queda por explorar el aspecto decisivo del problema, que es la estabilidad dinámica del equilibrio.

El problema se plantea en términos muy semejantes según se acepte la versión (1) o la versión (1-a) de la tasa de inmigración urbana. Limitaremos la discusión al segundo caso, en el que se tiene:

$$
\frac{d E}{E d t}=\frac{d N}{N d t}-\frac{d S}{S d t}=\gamma-\beta-F\left(\gamma x \frac{E}{1-E}-1\right)
$$

8 Ibid., p. 145.

9 Loc. cit. 


$$
\begin{gathered}
\frac{d}{d t}\left[\left(E^{* *}-E\right)^{2}\right]= \\
=-2\left(E^{* *}-E\right) \frac{d E}{d t}=-2\left(E^{* *}-E\right) E\left[\gamma-\beta-F\left(\gamma \times \frac{E}{1-E}-1\right)\right]
\end{gathered}
$$

La estabilidad global de la ecuación diferencial (9) quedará establecida afirmativamente si

$$
\frac{d}{d t}\left[\left(E^{* *}-E\right)^{2}\right]
$$

es siempre negativa, es decir, si la distancia entre la solución y el punto de equilibrio disminuye con el tiempo, para valores de la distancia diferentes de cero. ${ }^{10}$ Ahora bien, para $E^{* * *}>E$, el segundo miembro de (9) y por lo tanto el de (10) se hacen negativos, siempre y cuando $F$ sea una función monotónica creciente, que es lógico suponer. En efecto se tiene, de un lado,

$$
\frac{d}{d E}\left(\frac{E}{1-E}\right)=\frac{1}{(1-E)^{2}}>0
$$

Por otra parte, $\gamma-\beta-F\left(\gamma x \frac{E}{1-E}-1\right)$ se anula para $E=E^{* *}$, por definición de $E^{* *}$.

La monotonicidad de $F$ y (11) aseguran que la misma expresión será positiva para $E^{* *}>E$, con lo cual queda demostrado que

$$
\frac{d}{d t}\left[(E * *-E)^{2}\right]<0
$$

La demostración es idéntica si $E^{* *}<E$. El mismo resultado de estabilidad global se obtiene para la versión (1) de la tasa de inmigración urbana.

III. Dinámica urbana bajo el supuesto de atracción por parte del SeCTOR TRADICIONAL

Hasta ahora se ha trabajado con la hipótesis de que el ingreso esperado en la ciudad depende exclusivamente de las oportunidades de empleo y de ingreso que ofrece el sector moderno. Bajo esa hipótesis el sector tradicional constituye apenas un eslabón intermedio en el proceso de aculturación del migrante, sin ventajas propias con respecto a una situación de desempleo abierto, $\mathrm{y}$ mucho menos con respecto a las condiciones que predominan en las zonas rurales. Este tratamiento no es realista, porque olvida el hecho de que, quien está incorporado al sector urbano tradicional, percibe un modesto salario,

10 S. Karlin, Mathematical Methods and Theory in Games, Programming and Economics. Readings, Mass., Addison Wesley, 1959, p. 311, Vol. 1. 
frecuentemente reforzado por subsidios que la comunidad le ofrece en forma de diversas facilidades como educación, servicios públicos, acceso a centros de salud y otras amenidades de las cuales no disfruta ordinariamente el campesino. En términos teóricos, parece racional que al migrante en prospecto se le ofrecen dos alternativas en la ciudad. La primera con una probabilidad $\pi(t)$ de vincularse al sector moderno, en el cual percibe un ingreso $x Y_{r}$. La segunda, con una probabilidad $1-\pi(t)$ de acomodarse en el sector marginal, en donde dispondría de un ingreso real $z Y_{r},(x>z)$. Las consecuencias de introducir en el modelo el desempleo urbano abierto serán exploradas posteriormente.

En estas condiciones el ingreso esperado en la ciudad será $\pi(t) x Y_{r}+[1-\pi(t)] z Y_{r}$, de tal manera que tendremos las siguientes expresiones modificadas para la tasa de crecimiento de la fuerza laboral urbana y para el comportamiento dinámico de la variable $E$ :

$$
\begin{gathered}
\frac{d S}{S d t}=\beta+F[\pi(t)(x-z)+z-1] \\
\frac{d E}{E d t}=\frac{d N}{N d t}-\frac{d S}{S d t}=\gamma-\beta-F[\pi(t)(x-z)+z-1]
\end{gathered}
$$

Si se admite que sólo una fracción $k$ de los trabajadores urbanos subocupados aspiran y son elegibles para lograr una posición en el sector económico moderno, tendremos modificando la ecuación (3)

$$
\pi(t)=\frac{\gamma E(t)}{k[1-E(t)]}
$$

Reemplazando en (14) y haciendo $\frac{d E}{d t}=0$, se obtiene como solución de equilibrio

$$
E+=\frac{1+F^{-1}(\gamma-\beta)-z}{1+F^{-1}(\gamma-\beta)-z+\frac{\gamma}{k}(x-z)}
$$

siendo $F^{-1}$ la función inversa de $F . F^{-1}$ será definida y monovalente, dada la supuesta monotonicidad de $F$. Resulta fácil demostrar, por el mismo método seguido al final de la sección anterior, que si $0<E^{+}<1$ el equilibrio será estable en el sentido más amplio. Sin embargo, es preciso destacar el hecho de que la existencia misma del equilibrio depende, como condición necesaria y suficiente, de que se cumpla la desigualdad

$$
1+F^{-1}(\gamma-\beta)-z>0,
$$

Para demostrarlo, consideremos el valor $\pi^{+}$que resulta de reemplazar (16) en (15). Este valor anulará el segundo miembro de (14), con lo cual 


$$
\pi^{+}(x-z)=1+F^{-1}(\gamma-\beta)-z
$$

Es natural que si se están generando empleos a una tasa positiva por período dentro del sector urbano moderno, ninguna probabilidad de enganche puede ser nula ni, por supuesto, negativa, como sí lo es $\pi^{+}$en (18) si no se cumple la condición (17), toda vez que $x>z$. En estas condiciones, tendremos, dada la monotonicidad creciente de $F$, que la tasa de inmigración urbana $i_{t}$, en cualquier período $t$, cumplirá la relación

$$
i_{t}>i^{+}=F\left[\pi^{+}(x-z)+z-1\right]=\gamma-\beta
$$

cuando no se cumple (17). La tasa de crecimiento de la fuerza laboral urbana sería entonces en todo momento mayor que $\gamma$, con lo cual $E$ se haría cada vez menor, produciéndose un proceso explosivo de marginalidad urbana.

El fenómeno opuesto ocurriría cuando se cumple la relación

$$
1+F^{-1}(\gamma-\beta)>x .
$$

En tal caso la probabilidad de equilibrio $\pi^{+}$en (18) resultaría absurdamente mayor que la unidad. Con una probabilidad subjetiva real igual o menor que uno, la fuerza laboral urbana crecerá a una tasa menor que la del empleo en el sector moderno, y la variable $E$ se aproximaría gradualmente al valor de la unidad. A partir del punto en que $E$ alcanza el valor $\frac{k}{k+\gamma}$, la probabilidad $\pi(t)$ percibida por el migrante se igualaría a la unidad.

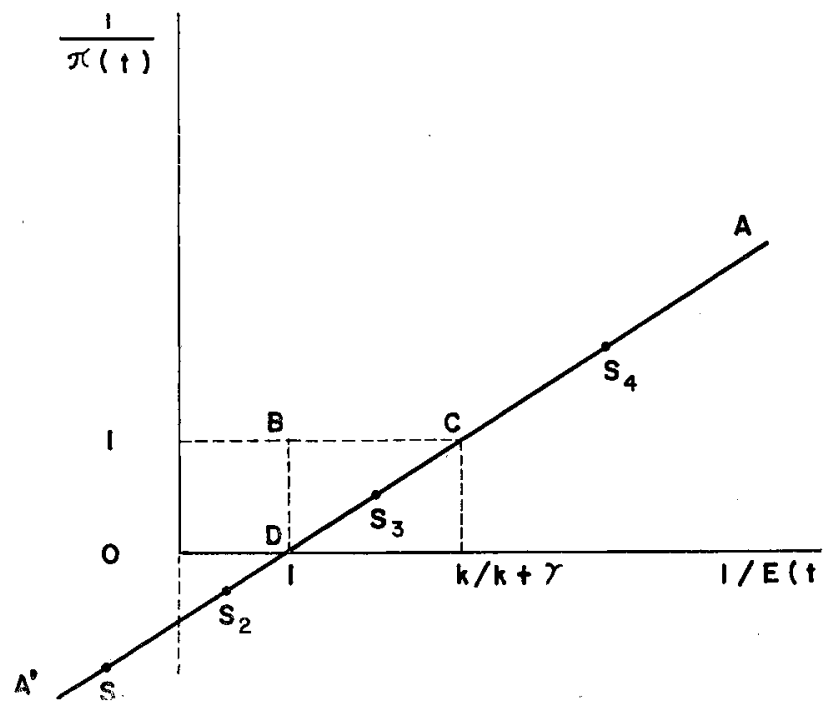


El argumento anterior puede expresarse por medio del diagrama 1, en donde se ilustra la relación entre $1 / \pi(t)$ y $1 / E(t)$ por medio de la función lineal, transformada de (15),

$$
\frac{1}{\pi(t)}=\frac{k}{\gamma}\left(\frac{1}{E}-1\right)
$$

Esta función está representada en el diagrama por la recta $A^{\prime} A$, en tanto que la línea poligonal $B C A, B=(1,1), C=\left(\frac{k}{k+\gamma}, 1\right)$ representa la relación factible entre $\pi(t)$ y $E(t)$.

En caso de no cumplirse la relación (17), las ecuaciones (16) y (18) suministrarán un punto espúreo de equilibrio como $S_{1}$ o $S_{2}$, en tanto que el sistema tenderá con el transcurso del tiempo hacia valores cada vez menores de $E(t)$ y $\pi(t)$. En cambio, en caso de que sea valedera la relación (20), también se satisfará la condición (17) puesto que $x>z$, y la solución de equilibrio, también espúrea, estará localizada en un punto intermedio entre $D$ y $C$, por ejemplo, $S_{3}$. El sistema tenderá realmente hacia $B$ con el transcurso del tiempo. Por último, si se cumple (17) pero no (20), el sistema tenderá hacia un punto de equilibrio, globalmente estable, como $S_{4}$, a la derecha de $C$.

$E l$ reconocimiento de fuerzas migratorias centrípetas ejercidas por el sector económico tradicional de la ciudad nos conduce así a una visión más compleja de la dinámica urbana y de las tendencias de la estructura del empleo. En particular, la existencia de un diferencial de ingresos tradicional urbano-rural suficientemente amplio sería un factor explicativo de la creciente marginalización experimentada en muchos centros urbanos de países en vía de desarrollo. Pueden citarse como ejemplos en América Latina los casos de Río de Janeiro y Lima, ciudades en donde existe evidencia indirecta de este proceso. ${ }^{11}$ Posiblemente Cali, entre otras ciudades colombianas, esté en las mismas circunstancias. Análogamente, en un reciente artículo de Charles Frank se comprueba el mismo fenómeno en un número significativo de países africanos, ${ }^{12}$ a pesar de que el autor expresa su creencia de que el sistema tenderá eventualmente hacia un estado de equilibrio. ${ }^{13}$

Es pertinente hacer algunas observaciones en torno a la condición de explosividad marginal, $1+F^{-1}(\gamma-\beta) \leqq z$. Primero, ella define un umbral de $z$ por encima del cual el valor esperado del ingreso urbano determina siempre una tasa de inmigración de tal magnitud que la fuerza laboral de la ciudad crece a una tasa más alta que el empleo del sector moderno. Es decir, la variable crítica del comportamiento explosivo del sistema es el diferencial del ingreso entre el sector tradi-

11. En Río de Janeiro habia en 1960 un $10.2 \%$ de la población total de la ciudad que habitaba en favelas, mientras en 1967 la proporción era de $18.9 \%$ (Anuario estadístico del Brasil, 1967). En Lima, la población de las barriadas, que en 1958 era del $10 \%$ de la total de la población de la ciudad, ascendía en 1964 al $20 \%$. H. L. Browning, "The Demography of the City", The Urban Explosion in Latin America, Glenn H. Beyer (compilador), Ithaca, N. Y., Cornell University Press, 1967, p. 101.

12 Op. cit., pp. 252-255.

13 Ibid., pp. 267-268. 
cional urbano y el sector rural. No importa qué tan alto sea $x$, el sistema tiende a una posición de equilibrio si $z$ no traspasa el valor crítico. En este sentido $x$ sólo tiene una influencia inversa sobre el valor de $E+$, cuando éste existe, pero no juega en el aspecto cualitativo de la dinámica urbana. Por consiguiente, parecería que una política de corrección de la explosividad debería apuntar hacia un mejoramiento relativo de las condiciones del campesino, tanto en términos de oportunidades de empleo como de ingreso real, con respecto al subempleado urbano. En cambio toda política de ampliación de servicios urbanos tendiente a cubrir las necesidades de la población marginal creciente, tendería a perpetuar la explosividad existente.

En segunda instancia, aunque el ingreso del sector moderno urbano no tiene una influencia directa sobre la existencia del equilibrio, sería irrealista olvidar que un alto nivel de $x$ puede presionar, a través de diversos mecanismos institucionales, la correspondiente alza de $z$, en vista de que ambos grupos de trabajadores coexisten en un mismo núcleo.

En tercer lugar, la sensibilidad de la existencia de un equilibrio a cambios en la tasa de generación de empleos o en la tasa de incremento natural de la fuerza de trabajo urbana es un asunto oscuro mientras no se esclarezca la naturaleza de la función $F$ mediante una medición empírica de las variables del modelo. Pero no es muy difícil imaginar situaciones en las que una dinámica explosiva requeriría para su eliminación cambios muy sustantivos en $\gamma$, o alternativamente, reducciones demasiado drásticas en $\beta$. Si por ejemplo se contempla la situación $z=1.30, \gamma=.04$ por año, $\beta=.03$ por año y $F(\alpha)=\frac{1}{10} \alpha$ dentro del intervalo de estudio, $z-1-F^{-1}(\gamma-\beta)=1.20$. Se requeriría elevar $\gamma$ por encima de .06 por año, lo que significa un aumento del $50 \%$ en el número anual de nuevos empleos en el sector moderno. $O$ alternativamente, sería necesaria una reducción de $\beta$ a nivel por debajo de .01 por año, para eliminar el problema de la explosividad marginal, subsistiendo sin embargo el problema de un valor muy bajo de equilibrio para $E$, que podría perpetuar la inestabilidad sociopolítica del sistema.

La efectividad de manipular la dinámica cualitativa del sistema a través de planes de control de natalidad urbana aparece aún más problemática si se tiene en cuenta que antes de un período de más de quince años esos planes no se traducirían en una disminución de la fuerza laboral, particularmente si al mismo tiempo se presenta un aumento en las tasas de participación femenina como consecuencia de menores cargas familiares.

\section{UN TRATAMIENTO ALTERNATIVO DE LA TASA DE INMIGRACIÓN}

Hasta ahora se ha considerado que el diferencial relativo urbanorural de ingresos esperados define la tasa de inmigración urbana, independientemente de la proporción que exista entre las poblaciones del campo y las de las ciudades. En principio no hay razón alguna para preferir este tratamiento a otro alternativo que considere que el 
mismo diferencial relativo de ingresos se expresa en una tasa de emigración rural en lugar de una tasa de inmigración urbana. No parece lógico pensar que, a medida que la población total del país se concentra cada vez más fuertemente en las ciudades, se presentará una estabilidad de la tasa de inmigración urbana para un diferencial dado de ingresos, tasa que significaría un flujo progresivamente más caudaloso y una tasa de emigración desde el campo aún más acelerada. En última instancia este fenómeno puede ser insostenible y podría esperarse una reducción en la tasa de inmigración urbana.

Por esta razón trataremos de explorar la dinámica de urbanización y de marginalidad que resulta de introducir la hipótesis alternativa

$$
e_{t}=G\left[\frac{Y_{u}(t)-Y_{r}}{Y_{r}}\right]
$$

en que $e_{t}$ es la tasa de emigración rural en el instante $t, G$ es una nueva función, $Y_{u}(t)$ mantiene su mismo significado anterior de ingreso esperado en la ciudad, y $Y_{r}$ es el ingreso real en el campo, supuesto invariable con el curso del tiempo. Si $S_{u}(t)$ y $S_{r}(t)$ representan las poblaciones urbana y rural respectivamente, la tasa de inmigración $i_{t}$ será:

$$
i_{t}=G\left[\frac{Y_{u}(t)-Y_{r}}{Y_{r}}\right] \frac{S_{r}(t)}{S_{u}(t)}
$$

En esta forma, con el proceso de urbanización, deberá esperarse, para un mismo diferencial de ingresos, un desplazamiento de $i_{t}$ en la forma ilustrada en el diagrama 2. A medida que la proporción $\frac{S_{r}(t)}{S_{u}(t)}$ disminuye, la función de migración $O A$ se corre hacia $O A^{\prime}, O A^{\prime \prime}$, etc.,

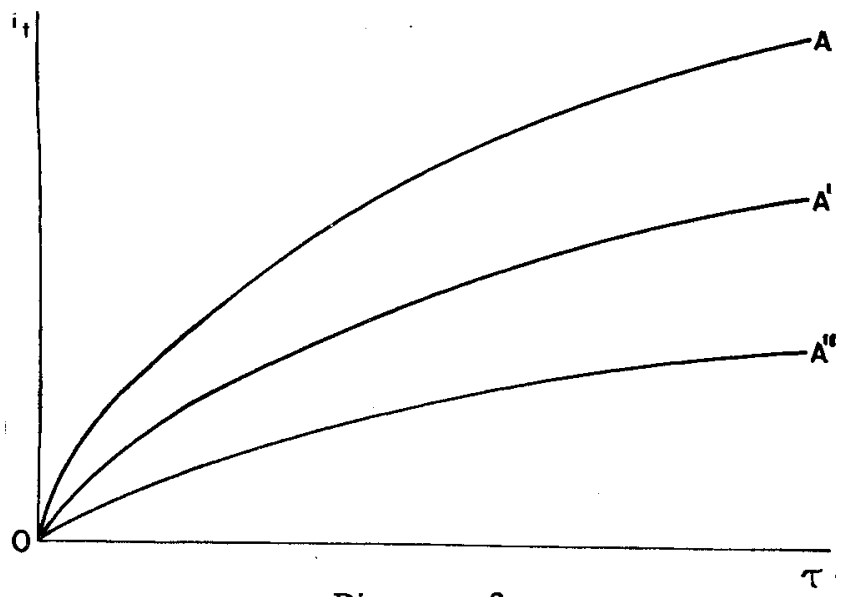


para un mismo valor $\tau$, que expresa el diferencial relativo de ingresos esperados entre la ciudad y el campo. Para explorar la dinámica del sistema, consideremos que la fuerza laboral urbana crece a una tasa vegetativa $\beta$, en tanto que la fuerza laboral rural crece a una tasa correspondiente $\beta^{\prime}$. Tendremos entonces,

$$
\begin{aligned}
& \frac{d S_{r}(t)}{S_{r}(t) d t}=\beta^{\prime}-G\left[\frac{Y_{u}(t)-Y_{r}}{Y_{r}}\right] \\
& \frac{d S_{u}(t)}{S_{u}(t) d t}=\beta+G\left[\frac{Y_{u}(t)-Y_{r}}{Y_{r}}\right] \frac{S_{r}(t)}{S_{u}(t)}
\end{aligned}
$$

Para que la variable $\frac{N_{t}}{S_{u}(t)}=E_{t}$ esté en una posición de equilibrio se requiere que $S_{u}(t)$ esté creciendo a una tasa constante $\gamma$, igual a la de generación del empleo en el sector moderno. La primera derivada del primer miembro de (25) deberá ser nula en tales condiciones. Entonces se tiene:

$G^{\prime}\left[\frac{Y_{u}(t)-Y_{r}}{Y_{r}}\right] \cdot \frac{S_{r}(t)}{S_{u}(t)} \cdot \frac{d}{d t}\left[\frac{Y_{u}(t)}{Y_{r}}\right]+G\left[\frac{Y_{u}(t)-Y_{r}}{Y_{r}}\right] \frac{d}{d t}\left[\frac{S_{r}(t)}{S_{u}(t)}\right]=0$

Ahora

$$
\frac{Y_{u}(t)}{Y_{r}}=\pi(t)(x-z)+z
$$

Además, según (15),

$$
\begin{aligned}
\frac{d}{d t}\left[\frac{Y_{u}(t)}{Y_{r}}\right] & =(x-z) \frac{d}{d t} \pi(t)=(x-z) \cdot \frac{\gamma}{k} \frac{d}{d t}\left[\frac{E_{t}}{1-E_{t}}\right] \\
\therefore \quad \frac{d}{d t}\left[\frac{Y_{u}(t)}{Y_{r}}\right] & =(x-z) \frac{\gamma}{k} \cdot \frac{1}{\left(1-E_{t}\right)^{2}} \cdot \frac{d E_{t}}{d t}
\end{aligned}
$$

Pero, en equilibrio $\frac{d E_{t}}{d t}=0$, con lo cual se simplifica (26).

$$
G\left[\frac{Y_{u}(t)-Y_{r}}{Y_{r}}\right] \cdot \frac{d}{d t}\left[\frac{S_{r}(t)}{S_{u}(t)}\right]=0
$$

Si de otro lado $\gamma>\beta$, la tasa de inmigración urbana de equilibrio es positiva, es decir,

$$
G\left[\frac{Y_{u}(t)-Y_{r}}{Y_{r}}\right]>0
$$


para dicha posición, y por consiguiente,

$$
\frac{d}{d t}\left[\frac{S_{r}(t)}{S_{u}(t)}\right]=0
$$

La ecuación (31) significa que el equilibrio intra-urbano en términos de la variable $E$ requiere como condición necesaria el equilibrio demográfico interregional, en términos de la proporción entre las poblaciones rural y urbana. Si llamamos $C$ el valor de equilibrio de $\frac{S_{r}(t)}{S_{u}(t)}$, tendremos, considerando la posición de estabilidad,

$$
\frac{\frac{d}{d t}\left(\frac{S_{r}}{S_{u}}\right)}{\frac{S_{r}}{S_{u}}}=\frac{d S_{r}}{S_{r} d t}-\frac{d S_{u}}{S_{u} d t}=0
$$

Reemplazando (24) y (25) en (32),

$$
\beta^{\prime}-G\left(\frac{Y_{u}}{Y_{r}}-1\right)_{\text {equilibrio }}-\beta-G\left(\frac{Y_{u}}{Y_{r}}-1\right) \text { equilibrio } \quad \mathrm{C}=0
$$

en donde $G\left(\frac{Y_{u}}{Y_{r}}-1\right)$ equilibrio es el valor de la función $G$ para la posición de estabilidad del sistema. Dicho valor es calculable mediante la ecuación (25)

$$
G\left(\frac{Y_{u}}{Y_{r}}-1\right) \text { equilibrio }=\frac{\gamma-\beta}{C}
$$

Reemplazando en (33),

$$
\begin{aligned}
& \beta^{\prime}-\frac{\gamma-\beta}{C}-\beta-\gamma+\beta=0 \\
& C=\frac{\gamma-\beta}{\beta^{\prime}-\gamma}
\end{aligned}
$$

Para que exista equilibrio debe tenerse $C>0$. Como se ha supuesto $\gamma>\beta$, se requerirá entonces $\beta^{\prime}>\gamma$. En otros términos, el sistema no podrá poseer un estado de equilibrio si no se satisface la condición de que la tasa de generación de empleo en el sector moderno de las ciudades tenga un valor intermedio entre las tasas vegetativas de incremento de las fuerzas laborales rural y urbana, debiendo ser esta última inferior a la primera. Mientras más próximo a $\beta$ sea $\gamma$ relativamente a la distancia entre $\beta$ y $\beta^{\prime}$, mayor será el grado de urbanización a que tiende la población. 
En términos generales, la condición $C>0$ no es suficiente para la existencia de un estado de equilibrio. Si se tiene

$$
1-z+G^{-1}\left(\beta^{\prime}-\gamma\right) \leqq 0
$$

aún con $\beta^{\prime}>\gamma$, no habrá equilibrio. Alternativamente puede ilustrarse este punto por medio de la solución del estado estable $E$ - que resulta

$$
E \cdot=\frac{1+G^{-1}\left(\beta^{\prime}-\gamma\right)-z}{1+G^{-1}\left(\beta^{\prime}-\gamma\right)-z+(x-z) \frac{\gamma}{k}}
$$

que no proporcionará estados factibles cuando el numerador es negativo.

Pasamos ahora a analizar más generalmente el problema de la estabilidad de $E$, en términos locales, es decir para perturbaciones muy pequeñas con respecto al equilibrio. Para este propósito enfocaremos un sistema de dos variables $x, y$, siendo

$$
x=\frac{S_{r}}{S_{u}}, \quad y=E
$$

De las ecuaciones (24) y (25) se deduce

$$
\frac{d x}{d t}=x[a-(1+x) g(y)]=f_{1}(x, y)
$$

en tanto que de (14) se obtiene

$$
\frac{d y}{d t}=y[b-x g(y)]=f_{2}(x, y)
$$

en donde

$$
a=\beta^{\prime}-\beta, \quad b=\gamma-\beta, \quad g(y)=G\left[\gamma(x-z) \frac{y}{1-y}+z-1\right]
$$

$\sigma(y)$ es naturalmente una función monotónicamente creciente y derivable.

El sistema (39) y (40) tiene como única solución factible de equilibrio

$$
x_{0}=\frac{b}{a-b}, \quad y_{0}=g^{-1}(a-b)
$$

Ya hemos visto que el sistema exige como condición de equilibrio (bajo la hipótesis de inmigración urbana $\gamma>\beta$ ), la desigualdad $\beta^{\prime}>\gamma>\beta$, es decir que $b>0,(a-b)>0$. 
Trasladando el sistema (39), (40) al punto $x_{0}, y_{0}$, se tiene, para pequeñas perturbaciones, el siguiente par de ecuaciones de variación ${ }^{14}$

$$
\begin{aligned}
& \frac{d x}{d t}=f_{1 x}\left(x_{0}, y_{0}\right) \Delta x+f_{1 y}\left(x_{0}, y_{0}\right) \Delta y \\
& \frac{d y}{d t}=f_{2 x}\left(x_{0}, y_{0}\right) \Delta x+f_{2 y}\left(x_{0}, y_{0}\right) \Delta y
\end{aligned}
$$

siendo $f_{1, m}$ la derivada parcial de la función 1 , con respecto a la variable $m$. Se tiene como condición necesaria y suficiente para la estabilidad del sistema, que las raíces de la ecuación característica en $p$ sean de signo negativo.15

$$
\left|\begin{array}{cc}
f_{1 x}-p & f_{1 y} \\
f_{2 x} & f_{2 y}-p
\end{array}\right|=0
$$

Ahora bien,

$$
\begin{aligned}
& f_{1 x}=-b, \quad f_{1 y}=\frac{a b}{(a-b)^{2}} g^{\prime}\left(y_{0}\right) \\
& f_{2 x}=-y_{0}(a-b), \quad f_{2 y}=-\frac{b}{a-b} y_{0} g^{\prime}\left(y_{0}\right)
\end{aligned}
$$

Reemplazando en (45)

$$
(b+p)\left[\frac{b}{a-b} y_{0} g^{\prime}\left(y_{0}\right)+p\right]-\frac{a b}{(a-b)} g^{\prime}\left(y_{0}\right) y_{0}=0
$$

o simplificando

$$
p^{2}+b p-y_{0} g^{\prime}\left(y_{0}\right)(a-b)=0
$$

Como $g$ es monotónicamente creciente, $g^{\prime}\left(y_{0}\right)>0$, además $y_{0}>0$, $a>b$, la ecuación (46) tendrá un término positivo en $p^{2}$ y un término independiente negativo, es decir sus dos raíces serán de signo contrario, lo cual demuestra la inestabilidad local del sistema en torno al punto de equilibrio.

14 Agradezco al profesor Germán Lemoine su cooperación en la solución de este problema.

15 Edouard Goursat, A Course in Mathematical Analysis, Vol. III, Parte uno, Dover Publications, 1964, pp. 3435. 
V. CONSIDERACIÓN DEL DESEMPLEO ABIERTO EN LAS CIUDADES

Intuitivamente, la existencia de desempleo en los centros urbanos debe atenuar las posibilidades de marginalidad explosiva en la medida en que sea captado y tenido en cuenta en las decisiones de migración. Este problema será explorado tentativamente en términos muy simplistas, a saber: supondremos, de un lado, que la persona que consigue un empleo dentro del sector moderno no está sujeta a riesgo alguno de perderlo. Por otra parte, se postulará una probabilidad constante $k^{\prime}$ de quedar desempleado para el contingente de la fuerza laboral urbana no incorporada al sector económico moderno. No entraremos a discutir aquí la pregunta espinosa, pero también muy importante, sobre las fuerzas que generan el desempleo dentro del sector tradicional. En realidad, estas fuerzas pueden representar una insuficiencia de absorción de empleo por parte de este último sector, o bien una incapacidad o insatisfacción de un segmento de la fuerza laboral marginada.

Bajo los supuestos anteriores el migrante potencial sopesaría las posibilidades de seguir percibiendo un ingreso $Y_{r}$ en el campo, contra tres alternativas: la primera, con una probabilidad $\pi(t)$, de engancharse al sector urbano moderno, con un ingreso $x Y_{r}$; la segunda, con una probabilidad $[1-\pi(t)] k^{\prime}$, de quedar desempleado en la ciudad en donde podría recibir un subsidio $z Y_{r}$; la tercera, con una prababilidad $[1-\pi(t)]\left(1-k^{\prime}\right)$ de incorporarse al sector tradicional urbano, con un ingreso $z Y_{r}$. Conservando el enfoque expuesto en la sección (3) tendríamos,

$$
\frac{d S}{S d t}=\beta+F\left[x \pi(t)+\left(1-\pi_{t}\right) k^{\prime} y+\left(1-k^{\prime}\right) z-1\right]
$$

El supuesto de equilibrio $E++$ del sistema para $\frac{d E}{d t}=0$, será

$$
E^{++}=\frac{1+F^{-1}(\gamma-\beta)+k^{\prime}(z-y)-z}{1+F^{-1}(\gamma-\beta)+k^{\prime}(z-y)-z+\frac{\hat{i}^{\prime}}{k} x-z+k^{\prime}(z-y)}
$$

y la condición de existencia será

$$
1+F^{-1}(\gamma-\beta)+k^{\prime}(z-y)-z>0
$$

Manteniéndose el mismo valor de $\gamma, \beta$ y $z$, la ecuación (49) será menos restrictiva que (17), pero aún subsiste el riesgo de explosividad marginal $\left(\frac{d E}{d t}<0\right.$, para todo valor de $\left.E\right)$. La tasa general de desempleo será $k^{\prime}\left(\frac{S-N}{S}\right)=k^{\prime}(1-E)$, es decir, tenderá a moverse en un sentido contrario a $E$. En caso de explosividad marginal, tendremos una tendencia creciente de desempleo abierto hacia el valor 
de $k$ '. Si se cumple la condición (45) el sistema tendrá estabilidad dinámica, con respecto al valor de equilibrio $E^{+}$deducido en (16), tendremos $E^{++}<E^{+}$.

\section{Dos Modelos DE DUALIDAD ECONÓMICA URBaNa}

En un reciente artículo, ${ }^{16}$ Nelson ha expuesto un modelo dualista de desarrollo industrial de acuerdo con el cual, dentro de cada rama manufacturera, las empresas de mayor productividad tienden a lo largo del tiempo a absorber una proporción creciente de la producción y del empleo totales correspondientes. Los resultados teóricos de este modelo fueron comprobados empíricamente por medio de las tendencias de industrialización observadas en Colombia durante los años 1958-1964, para una gama de veinte renglones manufactureros. Mientras en 1958 las firmas de más de 200 empleados representaban un $35 \%$ del volumen de ocupación, la proporción correspondiente en 1964 era del $65 \%$. Una tendencia semejante se observó con respecto a las cifras de valor agregado.17

A primera vista, este modelo dualista y su ilustración empírica podrían interpretarse como una evidencia contraria a las posibilidades de marginalidad creciente en los medios urbanos. La diferencia básica entre ambos modelos consiste en que, mientras por un lado dentro de una rama industrial existe competencia entre firmas de distinto grado de modernización, tanto en lo que se refiere al mercado de trabajo como al mercado de un mismo tipo de productos industriales, por otra parte entre el sector moderno urbano y el tradicional existe una gran gama de actividades dentro de las cuales no se presenta competencia significativa al nivel de mercado de productos o de servicio. Tal es el caso del servicio doméstico, de los servicios ambulantes y del pequeño comercio, que son típicos del sector tradicional. También es posible que predomine un mercado de competencia monopolística entre la pequeña artesanía y las grandes fábricas. Dadas estas condiciones, los resultados de ambos modelos resultan compatibles, particularmente cuando se consideran las siguientes hipótesis asentadas por Nelson:

a) La tasa de crecimiento de la producción dentro de cada sector de una misma rama manufacturera es proporcional a la utilidad industrial por unidad de producto.

b) El costo unitario en cada sector es igual al costo de las firmas marginales, más una proporción de las rentas diferenciales de cada firma, con respecto a un precio del producto supuesto igual para los sectores moderno y tradicional.

c) Las firmas más productivas pagan los factores de producción, capital y trabajo, a precios que varían en la misma proporción para ambos factores, con respecto a las firmas menos productivas.

d) Las funciones de producción son homogéneas de primer grado, de tal manera que un objetivo de minimización de costos, den-

16 R. Nelson, "A Diffusion Model of International Productivity Differences in Manufacturing Industry", American Economic Review, diciembre de 1968, particularmente pp. 1231-1235 y cuadro 4.

17 Op. cit., p. 1244. 
tro de una situación de competencia perfecta en el mercado de factores, implicará una función lineal de costos totales con respecto al costo del capital y del trabajo.

A partir de esas hipótesis puede demostrarse que la tasa de incremento de la producción del sector tecnológicamente más avanzado mantendrá una diferencia constante con respecto a la del sector con tecnología menos eficiente. Si, por otra parte, se acepta el supuesto de que las productividades de la mano de obra mantienen una misma relación para los dos sectores, para una combinación dada de factores de producción, se desprende que el volumen de empleo en el sector moderno será una proporción creciente del empleo total vinculado a la respectiva rama manufacturera.

En síntesis, los dos modelos de dualismo no son comparables, por cuanto Nelson explora el proceso de cambio dentro de una misma rama de actividad económica, mientras en este artículo se han analizado las tendencias de urbanización con dos sectores económicos cuyas actividades no coinciden.

\section{RESUMEN Y POSIBILIDADES DE INVESTIGACIÓN ADICIONAL.}

a) La probabilización de la tasa migratoria urbano-rural con respecto a las oportunidades de empleo que ofrece la ciudad abre vías interesantes de exploración teórica sobre los fenómenos de migración. Este tipo de análisis, introducido por Todaro, requiere no obstante un esfuerzo de cuantificación con respecto a la función migratoria, sin el cual el realismo del modelo resulta bastante oscuro.

b) No obstante, si se introducen algunas modificaciones adecuadas a la función migratoria y si particularmente se reconocen fuerzas de atracción migratoria por parte del sector urbano tradicional, pueden deducirse resultados teóricos que podrían constituir una explicación provisional de los fenómenos de creciente marginalización urbana que se observa en varios países. La introducción del desempleo abierto o la atenuación de las tasas de inmigración como consecuencia de un proceso de urbanización pronunciado no eliminan completamente las posibilidades de explosividad marginal.

c) Si al sector tradicional urbano no se le reconocen fuerzas de atracción migratoria, el proceso tiende hacia una posición de equilibrio estable, tanto para la función inmigratoria propuesta por Todaro, como para la función modificada escogida en este artículo. No obstante, al alterar el significado de los diferenciales de ingreso esperado y hacerlos incidir sobre la tasa de emigración rural, la atracción del sector tradicional deja de ser decisiva para la estabilidad del sistema, como es muy fácil de ver siguiendo el argumento de la sección III.

d) El tratamiento de las interrelaciones entre desempleo abierto y marginalidad urbana propuesto en la sección IV ha sido superficial, al suponer una tasa constante de desempleo en el sector tradicional urbano. Las tendencias coincidentes entre ambos fenómenos merecen explorarse dentro de un contexto teórico más elaborado.

e) En este artículo se han trazado algunos lineamientos sobre la dinámica urbana, suponiendo que los diferenciales de ingreso 
entre los tres sectores, rural, tradicional urbano y moderno urbano, son parámetros del sistema. Igual cosa ocurre con la tasa de generación de empleo en el tercer sector. Una línea interesante de investigación sería la formulación de un modelo dinámico en la economía que hiciese endógenas las mencionadas variables. 